TRANSACTIONS OF THE

AMERICAN MATHEMATICAL SOCIETY

Volume 358, Number 12, December 2006, Pages 5179-5192

S 0002-9947(06)03882-7

Article electronically published on July 25, 2006

\title{
ON THE VARIETY GENERATED BY ALL NILPOTENT LATTICE-ORDERED GROUPS
}

\author{
V. V. BLUDOV AND A. M. W. GLASS \\ To Valerie Kopytov on his sixty-fifth birthday
}

\begin{abstract}
In 1974, J. Martinez introduced the variety $\mathcal{W}$ of weakly Abelian lattice-ordered groups; it is defined by the identity
\end{abstract}

$$
x^{-1}(y \vee 1) x \vee(y \vee 1)^{2}=(y \vee 1)^{2} .
$$

We prove

Theorem A. There is a centre-by-metabelian weakly Abelian ordered group that does not belong to the variety of lattice-ordered groups generated by all nilpotent lattice-ordered groups.

This answers two questions of V. M. Kopytov.

We extend our techniques to show

Theorem B. The quasivariety generated by all nilpotent lattice-ordered groups is the same as the variety generated by all nilpotent lattice-ordered groups.

Our proof also gives a set of defining identities for this variety.

In contrast to Theorem $\mathrm{A}$ we show

Theorem C. Every Abelian-by-nilpotent weakly Abelian lattice-ordered group belongs to the variety of lattice-ordered groups generated by all nilpotent lattice-ordered groups.

\section{BASIC DEFINITIONS AND FACTS}

We will use the shorthand $\mathbb{Z}$ for the group of integers under addition with the usual ordering, $\mathbb{N}$ for the set of non-negative integers, and $\mathbb{R}$ for the additive group of real numbers with the usual ordering.

We will use standard group-theoretic notation, as in, e.g., 7 and 14. In particular, if $G$ is any group, we write $\zeta(G)$ for the centre of $G$; and if $f, g, h \in G$, we write $f^{g}$ for $g^{-1} f g$ and $[f, g]$ for $f^{-1} g^{-1} f g=f^{-1} f^{g}$; and $[f, g, h]$ as a shorthand for $[[f, g], h]$, etc. If $H$ and $K$ are subgroups of $G$, we write $[H, K]$ for the subgroup

Received by the editors December 27, 2003.

2000 Mathematics Subject Classification. Primary 06F15, 20F18, $20 \mathrm{~F} 12$.

Key words and phrases. Nilpotent group, residually torsion-free-nilpotent, variety, quasivariety, commutator calculus, lattice-ordered group, weakly Abelian.

The first author was supported by the Russian Foundation for Basic Research, grant no. 0301-00320.

(C)2006 American Mathematical Society Reverts to public domain 28 years from publication 
generated by $\{[h, k]: h \in H, k \in K\}$ and define the lower central series $\gamma_{m}(G)$ of $G$ inductively:

$$
\gamma_{1}(G)=G, \gamma_{m+1}(G)=\left[\gamma_{m}(G), G\right]
$$

(for all $m=1,2,3, \ldots$ ). Each $\gamma_{m}(G)$ is an invariant subgroup of $G$ (and hence a normal subgroup of $G$ ). Moreover, $G$ is nilpotent class $c$ if and only if $\gamma_{c+1}(G)=\{1\}$; i.e., if $\left[g_{1}, \ldots, g_{c+1}\right]=1$ for all $g_{1}, \ldots, g_{c+1} \in G$. Thus $G / \gamma_{c+1}(G)$ is nilpotent of class $c$ for all groups $G$. Throughout, let $I_{k}(G)$ be the isolator subgroup of $\gamma_{k}(G)$ in $G\left(k \in \mathbb{Z}_{+}\right)$, and $\Gamma(G)$ be $\bigcap_{k \in \mathbb{Z}_{+}} I_{k}(G)$.

If $\mathfrak{X}$ is a class of groups closed under isomorphisms, then a group $G$ is called residually $\mathfrak{X}$ if there is a family $\left\{N_{i}: i \in I\right\}$ of normal subgroups of $G$ with $\bigcap_{i \in I} N_{i}=\{1\}$ and each $G / N_{i} \in \mathfrak{X}$; i.e., $G$ is a subdirect product of the $\mathfrak{X}$-groups $G / N_{i}(i \in I)$.

A group equipped with lattice operations such that $x(y \vee z) t=x y t \vee x z t$ and $x(y \wedge z) t=x y t \wedge x z t$ for all $x, y, z, t$ is called a lattice-ordered group (or $\ell$-group, for short). If the lattice order is total (i.e., for any pair of elements $x \& y$, either $x \vee y=x$ or $x \vee y=y$ ), then the $\ell$-group is said to be an ordered group (or o-group for short). As usual, we write $x \leq y$ as a shorthand for either $x \vee y=y$ or $x \wedge y=x$.

Let $G$ be a partially ordered group (or p.o. group, for short), $G^{+}=\{g \in G: g \geq$ $1\}$ and $G_{+}=G^{+} \backslash\{1\}$. If $X$ is a subset of a group $G$, let $N_{G}(X)$ be the normal subsemigroup of $G$ generated by $X$. As is well known (see, e.g., [12, page 2]),

Lemma 1.1. $G$ can be made into a p.o. group with $G_{+}=N_{G}(X)$ if and only if $1 \notin N_{G}(X)$.

Recall [12, Theorems 3.1.5 and 3.1.7]

Lemma 1.2. If $G$ is a nilpotent or metabelian p.o. group, then the order can be extended so that $G$ becomes an o-group.

As is well known (see, e.g., 4, Chapter 2]), every element of an $\ell$-group $G$ can be written in the form $f^{-1}$ for some $f, g \in G^{+}$; moreover, $\{|g|: g \in G\}=G^{+}=$ $\{g \vee 1: g \in G\}$ where we write $|g|$ for $g \vee g^{-1}$. Therefore $G^{+}$completely determines the order on $G$.

A subgroup $C$ of an o-group $G$ is called convex if for all $c_{1}, c_{2} \in C, g \in G$, $\left(c_{1} \leq g \leq c_{2}\right.$ implies $\left.g \in C\right)$. The set of convex subgroups of an o-group is totally ordered by inclusion [4, Lemma 3.1.2]. The set is closed under unions and intersections. Hence given any non-identity element $g$ of an o-group $G$, there is a unique subgroup $C_{g}$ maximal with respect to not containing $g$. It is called the value of $g$ and is strictly contained in the convex subgroup $C(g)$ of $G$ generated by $g$. Furthermore, $C_{g} \triangleleft C(g)$. The pair $\left(C_{g}, C(g)\right)$ is called a convex jump, and $C(g) / C_{g}$ is isomorphic (as an o-group) to an additive subgroup of $\mathbb{R}$ (see, e.g., 4, Chapters 3 and 4$]$ ).

As is also standard, we write $f \ll g$ for $f^{n} \leq|g|$ for all $n \in \mathbb{Z}$ (and say that $f$ is very much less than $g$ ). In any o-group, $f_{1} f_{2} \ll g$ whenever $f_{1} \ll g \& f_{2} \ll g$ (see, e.g., [4, Chapter 3]).

Residually ordered $\ell$-groups form a variety $\mathcal{R}$ in the class of all $\ell$-groups (see, e.g., [4, Section 3.8] or [9, Section 9.3]). Hence any $G \in \mathcal{R}$ is $\ell$-isomorphic to a subdirect product of o-groups. So any subvariety of $\mathcal{R}$ is generated by all of its o-groups. But any o-group is o-isomorphic to a subgroup of an ultraproduct of its 
finitely generated subgroups. Consequently we get:

Lemma 1.3. Any subvariety of $\mathcal{R}$ is generated by its finitely generated o-groups.

This is one of the essential tools in our proofs.

A lattice-ordered group $G$ is called weakly Abelian if $g^{-1}|f| g \leq|f|^{2}$ for all $f, g \in G$ 11] (or see [4, Section 6.4] or [9, Sections 6.2 and 9.4]). Equivalently, with the above shorthand, this law can be written in the form

$$
f^{g} \leq f^{2} \text { for all } f \in G^{+}, g \in G .
$$

Throughout we will use the following well-known result [11] (or see, e.g., [4, Lemma 6.4.1]):

Lemma 1.4. An $\ell$-group $G$ is weakly Abelian if and only if $|[f, g]| \ll|f|$ for all $f, g \in G$.

Hence every weakly Abelian $\ell$-group is residually ordered; i.e., a subdirect product of (weakly Abelian) o-groups [ibid.]. Weakly Abelian o-groups are called centrally ordered and have recently been studied by the authors with A. H. Rhemtulla (see [2] and [3]). Note that in any centrally ordered group, $C(g)$ and $C_{g}$ are normal subgroups of $G$ for all $g \in G \backslash\{1\}$. Moreover, $C_{g} g$ is central in $G / C_{g}$ and $[G, C(g)] \subseteq C_{g}[$ op. cit. $]$.

We will need one fact from [3]:

Lemma 1.5 ([3, Theorem D]). If $G$ is a finitely generated centrally ordered Abelianby-nilpotent group and $g$ is a non-identity element of $G$, then $G / C_{g}$ is residually torsion-free-nilpotent.

Any locally nilpotent $\ell$-group is weakly Abelian ([8, [13, or see [4, Theorem 6.D] or [9. Theorem 9.4.1]); so the variety of $\ell$-groups generated by all nilpotent $\ell$-groups is contained in $\mathcal{W}$. In 1984, V. M. Kopytov asked if the converse were true [16, Question 40] (cf., Kopytov's stronger question [10, Problem 5.23]).

Since weakly Abelian $\ell$-groups are residually ordered, the question is equivalent to:

Does every centrally ordered group belong to the variety of latticeordered groups generated by all nilpotent lattice-ordered groups?

Theorem A provides negative answers to Kopytov's questions.

For prior work on this topic, see [5] and [6].

Let $\mathfrak{N}$ be the class of all lattice-ordered groups that are nilpotent.

Throughout, let $\hat{\mathfrak{N}}$ denote the variety of lattice-ordered groups generated by $\mathfrak{N}$. So $\hat{\mathfrak{N}}$ is defined by all identities that hold in all nilpotent $\ell$-groups. Every element of $\hat{\mathfrak{N}}$ is an $\ell$-homomorphic image of a subdirect product of nilpotent o-groups.

We will write $q(\mathfrak{N})$ for the quasi-variety of lattice-ordered groups generated by $\mathfrak{N}$. It is the smallest class of $\ell$-groups that is closed under $\ell$-isomorphisms, sublattice subgroups, direct products and ultraproducts and contains $\mathfrak{N}$. It is the class of $\ell$-groups defined by all implications of the form

$$
\begin{gathered}
\left(\forall x_{1}, \ldots, x_{n}\right)\left[\left(u_{1}\left(x_{1}, \ldots, x_{n}\right)=1 \& \ldots \& u_{k}\left(x_{1}, \ldots, x_{n}\right)=1\right)\right. \\
\left.\rightarrow w\left(x_{1}, \ldots, x_{n}\right)=1\right]
\end{gathered}
$$

that hold in all $\ell$-groups belonging to $\mathfrak{N}$, where $u_{1}\left(x_{1}, \ldots, x_{n}\right), \ldots, u_{k}\left(x_{1}, \ldots, x_{n}\right)$, $w\left(x_{1}, \ldots, x_{n}\right)$ are $\ell$-group words.

Clearly, $q(\mathfrak{N}) \subseteq \hat{\mathfrak{N}}$. 


\section{The proof of Theorem A}

Let $G$ be a group with generators $a_{1}, a_{2}, y, c$ and relations

$$
\begin{gathered}
{\left[a_{1}, a_{2}\right]=\left[a_{1}, y\right]=\left[a_{2}, y\right]=1,} \\
{\left[a_{i}^{ \pm 1}, c, c\right]=\left[a_{i}^{2}, c, c\right]=1 \quad(i=1,2),} \\
c c^{a_{2}^{2}}=c^{2 y} \\
{\left[\left[a_{1}^{2}, c\right],\left[a_{2}, c\right]\right]=\left[\left[a_{1}^{2}, c\right],\left[a_{2}^{-1}, c\right]\right],} \\
{\left[d, a_{1}\right]=\left[d, a_{2}\right]=[d, y]=[d, c]=1,}
\end{gathered}
$$

where $d:=\left[\left[a_{1}^{2}, c\right],\left[a_{2}, c\right]\right]$.

This is a finitely presented group. By (5),

$$
d \in \zeta(G) .
$$

To prove Theorem $\mathrm{A}$, we first establish two facts:

(i) if $G^{\psi}$ is a torsion-free nilpotent homomorphic image of $G$, then $d^{\psi}=1$ (and so $d \in \Gamma(G))$;

(ii) $G$ has a centrally orderable homomorphic image $G^{\varphi}$ with $d^{\varphi} \neq 1$.

To prove (i) we need

Lemma 2.1. In $G$,

$$
c \in \zeta\left(\left\langle c, c^{\left\langle a_{1}^{2}\right\rangle}, c^{\left\langle a_{2}^{ \pm 1}\right\rangle}\right\rangle\right),
$$

and for all $m \in \mathbb{Z}_{+}$,

$$
\left[\left[a_{1}^{2}, c\right],\left[a_{2}^{2 m+1}, c\right]\right]=d .
$$

Proof. Trivially, in any group $L$,

$$
[x, y]=1 \text { implies }\left[x^{p}, y^{q}\right]=1 \text { for all } p, q \in \mathbb{Z} .
$$

By standard commutator calculus and induction on $k$,

$$
[x, y, y]=1 \text { implies }\left[x, y^{k}\right]=[x, y]^{k} \text { for all } k \in \mathbb{Z} .
$$

Moreover,

$$
[x, y, y]=\left[y^{-x} y, y\right]=\left[y^{-x}, y\right]^{y} .
$$

Thus

$$
[x, y, y]=1 \text { if and only if }\left[y^{x}, y\right]=1 \text {. }
$$

This gives (7).

We prove (8) by induction on $m \in N \cup\{-1\}$.

By (44), $d=\left[\left[a_{1}^{2}, c\right],\left[a_{2}^{-1}, c\right]\right]$, so (8) is true if $m=-1$ or 0 .

We now induct on $m$ using $y$.

We have $d=\left[c^{a_{1}^{2}}, c^{a_{2}}\right]=\left[c^{a_{1}^{2}}, c^{a_{2}^{-1}}\right]$.

Assume that

$$
d^{4}=\left[c^{2 a_{1}^{2}}, c^{2 a_{2}^{2 k+1}}\right]=\left[c^{2 a_{1}^{2}}, c^{2 a_{2}^{2 k-1}}\right] .
$$


Then

$$
\begin{aligned}
d^{4} & =\left[c^{2 a_{1}^{2}}, c^{2 a_{2}^{2 k+1}}\right]^{y}=\left[c^{a_{1}^{2}} c^{a_{1}^{2} a_{2}^{2}}, c^{a_{2}^{2 k+1}} c^{a_{2}^{2 k+3}}\right] \\
& =\left[c^{a_{1}^{2}}, c^{a_{2}^{2 k+1}} c^{a_{2}^{2 k+3}}\right]^{c_{1}^{2} a_{2}^{2}} \cdot\left[c^{a_{1}^{2} a_{2}^{2}}, c^{a_{2}^{2 k+1}} c^{a_{2}^{2 k+3}}\right] \\
& =\left[c^{a_{1}^{2}}, c^{a_{2}^{2 k+3}}\right]^{c_{1}^{a_{1}^{2} a_{2}^{2}}} \cdot\left[c^{a_{1}^{2}}, c^{a_{2}^{2 k+1}}\right]^{c_{2}^{a_{2}^{2 k+3}}} c^{a_{1}^{2} a_{2}^{2}} \cdot\left[c^{a_{1}^{2} a_{2}^{2}}, c^{a_{2}^{2 k+3}}\right] \cdot\left[c^{a_{1}^{2} a_{2}^{2}}, c^{a_{2}^{2 k+1}}\right]^{c_{2}^{a_{2}^{2 k+3}}} \\
& =\left[c^{a_{1}^{2}}, c^{a_{2}^{2 k+3}}\right]^{c_{1}^{a_{1}^{2} a_{2}^{2}}} \cdot d^{3} .
\end{aligned}
$$

Thus, $\left[c^{a_{1}^{2}}, c^{a_{2}^{2 k+3}}\right]^{a_{1}^{2} a_{2}^{2}} \in \zeta(H)$ and $\left[c^{a_{1}^{2}}, c^{a_{2}^{2 k+3}}\right]=d$. So $\left[c^{a_{1}^{2}}, c^{a_{2}^{n}}\right]=d$ for all odd $n \in$ $\mathbb{Z}_{+}$. But $\left[\left[a_{1}^{2}, c\right],\left[a_{2}^{n}, c\right]\right]=\left[c^{a_{1}^{2}}, c^{a_{2}^{n}}\right]$ using (7) and standard commutator calculus. (8) now follows.

We now use Lemma 2.1 to establish (i).

Let $p$ be an odd prime and $2 m+1$ range over all powers of $p$ in (8). By Gruenberg's Theorem [14, Theorem 5.2.21], every finitely generated torsion-free nilpotent group is a residually finite $p$-group. By (8), $d=1$ in any finite $p$-group that is an image of $G$. Therefore, $d=1$ in every torsion-free nilpotent homomorphic image of $G$.

We now prove (ii). That is, the initial group, $G$, has a centrally ordered homomorphic image $G^{\varphi}$ with $d^{\varphi}=\left[\left[a_{1}^{2}, c\right],\left[a_{2}, c\right]\right]^{\varphi} \neq 1$. For this we construct an example. The first examples we considered were based on [1, Statement 6]; what is presented here is several modifications later.

Example 2.2. Let $C$ be the free nilpotent class 2 group with free generators $\left\{c_{m, n}: m, n \in \mathbb{Z}\right\}$. The centre of $C$ is the free Abelian group with free generators $\left[c_{m, n}, c_{p, q}\right](m, n, p, q \in \mathbb{Z})$ where $(m, n)>(p, q)$ lexicographically. We embed $C$ in a divisible nilpotent class 2 group $C^{b}$; so each element of $C^{b}$ can be written uniquely (to within the order that the commutators appear) in the form

$$
c_{m_{1}, n_{1}}^{r_{1}} \ldots c_{m_{k}, n_{k}}^{r_{k}} \prod_{i=1}^{\ell}\left[c_{p_{i}, q_{i}}, c_{p_{i}^{\prime}, q_{i}^{\prime}}\right]^{t_{i}},
$$

where $\left(m_{1}, n_{1}\right)>\cdots>\left(m_{k}, n_{k}\right)$ lexicographically, $p_{i}, p_{i}^{\prime}, q_{i}, q_{i}^{\prime} \in \mathbb{Z}$ with $\left(p_{i}, q_{i}\right)>$ $\left(p_{i}^{\prime}, q_{i}^{\prime}\right)$ lexicographically $(i=1, \ldots, \ell)$ and $r_{1}, \ldots, r_{k}, t_{1}, \ldots, t_{\ell} \in \mathbb{Q}$ (see [15, Theorem 8.5]). We add the relations

$$
\begin{gathered}
{\left[c_{m, n}, c_{p, q}\right]=\left[c_{m-p, 0}, c_{0, q-n}\right] \quad(m, n, p, q \in \mathbb{Z}),} \\
{\left[c_{m, 0}, c_{0,2 q}\right]=\left[c_{2 m+1,0}, c_{0,2 q+1}\right]=1 \quad(m, q \in \mathbb{Z}),} \\
{\left[c_{2 m, 0}, c_{0,2 q+1}\right]=\left[c_{2,0}, c_{0,1}\right]^{m} \quad(m, q \in \mathbb{Z}),}
\end{gathered}
$$

and quotient out from $C^{b}$ the divisible normal subgroup determined by these relations. We obtain a factor group $C^{\#}$ with centre $D$, the divisible closure of $\langle d\rangle$ in $C^{\#}$, where $d:=\left[c_{2,0}, c_{0,1}\right]$. So $D$ is a rank 1 Abelian group.

Note that $C^{\#}$ is a divisible nilpotent class 2 group and each element of $C^{\#}$ can be uniquely written in the form

$$
c_{m_{1}, n_{1}}^{r_{1}} \ldots c_{m_{k}, n_{k}}^{r_{k}} d^{t},
$$

where $\left(m_{1}, n_{1}\right)>\cdots>\left(m_{k}, n_{k}\right)$ lexicographically and $r_{1}, \ldots, r_{k}, t \in \mathbb{Q}$ (see [15, Theorem 8.5]). 
Let $a, b$ be automorphisms of $C^{b}$ determined by

$$
a: c_{m, n} \mapsto c_{m+1, n} \quad b: c_{m, n} \mapsto c_{m, n+1} .
$$

Note that $a, b$ induce automorphisms of $C^{\#}$.

Proof. We need only show that $a, b$ respect the relations (12)-(14). This is immediate since

$$
\left[c_{m, 0}, c_{0, q}\right]^{a}=\left[c_{m+1,0}, c_{1, q}\right]=\left[c_{m, 0}, c_{0, q}\right]=\left[c_{m, 1}, c_{0, q+1}\right]=\left[c_{m, 0}, c_{0, q}\right]^{b} .
$$

Therefore $a$ and $b$ induce well-defined automorphisms of $C^{\#}$.

We observe that automorphisms $a$ and $b$ commute and fix $D$ pointwise.

So we can construct a splitting extension $K=C^{\#} \rtimes\langle a, b\rangle$ with Abelian top group $\langle a, b\rangle$. Note that $\left.K / D \cong\left(C^{\#} / D\right)\right\rangle\langle a, b\rangle$ and $C^{\#} / D$ is Abelian. Thus $K / D$ is metabelian with torsion-free Abelian quotients $\gamma_{n}(K / D) / \gamma_{n+1}(K / D)$ and so is residually torsion-free-nilpotent. Furthermore, $\zeta(K)=D$.

Each element of $K$ can be written uniquely in normal form

$$
a^{p} b^{q} c_{m_{1}, n_{1}}^{r_{1}} \ldots c_{m_{k}, n_{k}}^{r_{k}} d^{t},
$$

where $p, q, m_{1}, n_{1}, \ldots, m_{k}, n_{k} \in \mathbb{Z}$ and $r_{1}, \ldots, r_{k}, t \in \mathbb{Q}$, and $\left(m_{1}, n_{1}\right)>\cdots>$ $\left(m_{k}, n_{k}\right)$ in the lexicographic order on $\mathbb{Z} \times \mathbb{Z}$.

Let $L=\left\langle a, b, c_{0,0}\right\rangle$. Now $d=\left[c_{2,0},\left[c_{0,0}, b^{2 m+1}\right]\right]$, so by applying Gruenberg's Theorem to $L / I_{n}(L)$, we deduce that $d \in I_{n}(L)$ for all $n \in \mathbb{Z}_{+}$. Hence $d \in$ $\Gamma(L) \subseteq \Gamma(K)$, whence $D \subseteq \Gamma(K)$. Since $\Gamma(K / D)=1$, we have $\Gamma(K) \subseteq D$. Therefore $D=\Gamma(K)$. Since $C^{\#}$ is divisible and $\left[C^{\#}, C^{\#}\right] \leq \zeta\left(C^{\#}\right)=D$, by the normal form for elements of $K$ we obtain $D=\Gamma(K)=\bigcap_{i=1}^{\infty} \gamma_{i}(K)$. It follows that $\gamma_{n}(K) / \gamma_{n+1}(K) \cong \gamma_{n}(K / D) / \gamma_{n+1}(K / D)$ for all $n \in \mathbb{Z}_{+}$. Thus $K$ has a central order with a series of convex subgroups

$$
\{1\}<D=\bigcap_{i=1}^{\infty} \gamma_{i}(K)<\cdots<\gamma_{n+1}(K)<\gamma_{n}(K)<\cdots<\gamma_{1}(K)=K .
$$

By (12)-(14), for all $m, q \in \mathbb{Z}$ we get (in $C^{\#}$ )

$$
\left[c_{m+2,0}, c_{0, q}\right]\left[c_{m-2,0}, c_{0, q}\right]=\left[c_{m, 0}, c_{0, q}\right]^{2}=\left[c_{m, 0}, c_{0, q+2}\right]\left[c_{m, 0}, c_{0, q-2}\right] .
$$

In $C^{\#}, c_{m, n}$ commutes with $c_{m+2, n}$ and $c_{m, n+2}(m, n \in \mathbb{Z})$. These lead us to the following:

Consider the injective endomorphism $\beta$ of $C^{b}$ determined by

$$
\beta: c_{m, n} \mapsto c_{m, n+2}^{1 / 2} c_{m, n}^{1 / 2} .
$$

A tedious but thoroughly routine verification shows that $\beta$ respects the relations (12)-(14). Thus $\beta$ induces an injective endomorphism of $C^{\#}$.

We extend $\beta$ to an injective endomorphism of $K$ by

$$
\beta: a \mapsto a ; \quad \beta: b \mapsto b .
$$

Let $K^{b}=\bigcup\left\{K^{\beta^{-n}}: n \in \mathbb{Z}_{+}\right\}$with central order inherited from $K$, and let $H=\left\langle K^{b}, \beta\right.$ : (17), (18) $\rangle$ be the ascending HNN-extension of $K$ with respect to $\beta$. By the normal form for HNN-extensions, we see that $H / D$ is a metabelian residually torsion-free-nilpotent group and $D=\zeta(H)$. Equations (17) and (18) show that $\beta$ acts as the identity on $D$, and also on each $\gamma_{n}(K) / \gamma_{n+1}(K)\left(n \in \mathbb{Z}_{+}\right)$. The latter follows at once from $c_{m, n}^{r \beta}=c_{m, n}^{r}\left[c_{m, n}^{r / 2}, b^{2}\right]$. Hence the central order on 
$K$ is preserved by the injective endomorphism $\beta$, and we can extend this central order to $H$ by the spelling for HNN-extensions, with

$$
K^{b} \ll \beta .
$$

Let $K_{0}$ be the subgroup of $H$ generated by $a, b, c_{0,0}, \beta$. Then $K_{0}$, the "Kopytov group", is centre-by-metabelian. By construction, the relations (1)-(5) hold in $K_{0}$ under the substitution $a, b, \beta, c_{0,0}$ for $a_{1}, a_{2}, y, c$, respectively, and $d \neq 1$ by the normal form of the HNN-extension. Moreover, $K_{0}$ is centrally orderable since $H$ is.

Thus, $K_{0}$ is a centrally orderable homomorphic image of $G$ satisfying (ii).

We must now show that (i) and (ii) imply Theorem $\mathrm{A}$. This follows immediately from the following more general theorem.

Theorem D. Let $G_{0}$ be a lattice-ordered group and $G_{0} \in \hat{\mathfrak{N}}$. If there is a group homomorphism $\varphi$ from a finitely presented group $G$ into $G_{0}$, then $\Gamma(G) \subseteq \operatorname{ker}(\varphi)$.

For if $G$ is defined by (11)-(5) and $K_{0}$ is the centrally ordered group constructed in Example 2.2. then it follows from (i) and (ii) that $d \in \Gamma(G) \backslash \operatorname{ker}(\varphi)$ where $G^{\varphi}=K_{0}$; hence $K_{0} \in \mathcal{W} \backslash \hat{\mathfrak{N}}$ and Theorem $\AA$ is established.

To prove Theorem D we will transform certain types of group implications into $\ell$-group identities. To achieve this we will need two lemmata.

Let $H$ be a finitely generated group with generators $h_{1}, \ldots, h_{n}$. Let $U=$ $\left\langle u_{1}, \ldots, u_{k}\right\rangle$ be a finitely generated subgroup of $H$. We write

$$
\Gamma_{U}(H)=\bigcap_{j=1}^{\infty} I\left(U^{H} \gamma_{j}(H)\right),
$$

where $I(G)$ is the isolator of $G$. So $\Gamma_{\{1\}}(H)=\Gamma(H)$. By construction,

$$
\{1\} \subseteq U \subseteq U^{H} \subseteq \Gamma_{U}(H) \subseteq H .
$$

Lemma 2.3. Let $H$ be a finitely generated group with generators $h_{1}, \ldots, h_{n}$, and let $U=\left\langle u_{1}, \ldots, u_{k}\right\rangle$ be a finitely generated subgroup of $H$. Then for any $w \in \Gamma_{U}(H)$, there are $m_{0}, \ldots, m_{k} \in \mathbb{Z}$ with $m_{0} \in \mathbb{Z}_{+}$such that

$$
w^{m_{0}} u_{1}^{m_{1}} \cdots u_{k}^{m_{k}} \in \Gamma_{[U, H]}(H) .
$$

Lemma 2.4. Let $F=F\left(x_{1}, \ldots, x_{n}\right)$ be the free group and $U=\left\langle u_{1}, \ldots, u_{k}\right\rangle$ a finitely generated subgroup of $F$. If $w \in \Gamma_{[U, F]}(F)$, then

$$
\left|w\left(y_{1}, \ldots, y_{n}\right)\right| \ll \bigvee_{j=1}^{k}\left|u_{j}\left(y_{1}, \ldots, y_{n}\right)\right|
$$

holds in every nilpotent lattice-ordered group.

Actually, $(* *)$ is an infinite set of identities since $w_{1} \ll w_{2}$ is a shorthand for $w_{1}^{m} \leq w_{2}$ for all $m \in \mathbb{Z}$.

We first show that the lemmata indeed imply Theorem D

Proof. Let $G=\left\langle g_{1}, \ldots, g_{n}: u_{1}\left(g_{1}, \ldots, g_{n}\right)=1, \ldots, u_{k}\left(g_{1}, \ldots, g_{n}\right)=1\right\rangle$. Let $F$ be the free group on $x_{1}, \ldots, x_{n}$ and $u_{j}=u_{j}\left(x_{1}, \ldots, x_{n}\right)$ be the result of replacing each occurrence of $g_{i}$ in $u_{j}\left(g_{1}, \ldots, g_{n}\right)$ by $x_{i}(1 \leq i \leq n ; 1 \leq j \leq k)$. Then the natural homomorphism $\psi: F \rightarrow G$ determined by $x_{i} \mapsto g_{i}(1 \leq i \leq n)$ has kernel $U^{F}$ where 
$U=\left\langle u_{1}, \ldots, u_{k}\right\rangle$. If $w\left(g_{1}, \ldots, g_{n}\right) \in \Gamma(G) \backslash \operatorname{ker}(\varphi)$, then $w\left(g_{1}, \ldots, g_{n}\right)^{\varphi} \neq 1$ in $G_{0}$. But $w\left(x_{1}, \ldots, x_{n}\right) \in \Gamma_{U}(F)$ by definition. By Lemma 2.3, there are $m_{0}, \ldots, m_{k} \in \mathbb{Z}$ such that

By Lemma 2.4.

$$
\hat{w}:=w^{m_{0}} u_{1}^{m_{1}} \cdots u_{k}^{m_{k}} \in \Gamma_{[U, F]}(F) .
$$

$$
1 \neq\left|w\left(g_{1}, \ldots, g_{n}\right)^{\varphi}\right|^{m_{0}}=\left|\hat{w}\left(g_{1}, \ldots, g_{n}\right)^{\varphi}\right| \ll \bigvee_{j=1}^{k}\left|u_{j}\left(g_{1}, \ldots, g_{n}\right)^{\varphi}\right|=1,
$$

since $u_{1}\left(x_{1}, \ldots, x_{n}\right), \ldots, u_{k}\left(x_{1}, \ldots, x_{n}\right) \in \operatorname{ker}(\varphi)$. This contradiction establishes the theorem.

We now prove Lemma 2.3 .

Proof. Let $w \in \Gamma_{U}(H)$. Then for every $j \in \mathbb{Z}_{+}$, there is a positive integer $t_{j}$ such that $w^{t_{j}} \in U^{H} \gamma_{j}(H)$; say,

$$
w^{t_{j}} \equiv u_{1}^{m_{1, j}} \cdots u_{k}^{m_{k, j}} \quad\left(\bmod [U, H]^{H} \gamma_{j}(H)\right)
$$

with $m_{1, j}, \ldots, m_{k, j} \in \mathbb{Z}$. We call $\left(*_{j}\right)$ a $j^{\text {th }}$-representation for $w$; it is not usually unique.

If $t_{j}^{\prime} \in \mathbb{Z}_{+}$and $m_{1, j}^{\prime}, \ldots, m_{k, j}^{\prime} \in \mathbb{Z}$ with

$$
\left(w^{\prime}\right)^{t_{j}^{\prime}} \equiv u_{1}^{m_{1, j}^{\prime}} \cdots u_{k}^{m_{k, j}^{\prime}} \quad\left(\bmod [U, H]^{H} \gamma_{j}(H)\right),
$$

then we say that the $\left(*_{j}\right)$ representation is less than the $\left(*_{j}^{\prime}\right)$ representation if

$$
\left(\left|m_{1, i}\right|, \ldots,\left|m_{k, j}\right|\right)<\left(\left|m_{1, i}^{\prime}\right|, \ldots,\left|m_{k, j}^{\prime}\right|\right),
$$

in the lexicographic ordering on $\mathbb{N}^{k}$. This is a well-ordering.

For each $j \in \mathbb{Z}_{+}$, among all the representations $\left(*_{j}\right)$ for positive powers of $w$, choose one so that the right-hand side of $\left(*_{j}\right)$ is minimal. We will assume that the sequence $\left\{t_{j}: j \in \mathbb{Z}_{+}\right\}$has been chosen so that, for each $j \in \mathbb{Z}_{+},\left(*_{j}\right)$ is minimal for all positive powers of $w$.

Fix $j_{1}$ and let $j \geq j_{1}$. We first establish:

Claim: If $m_{1, j_{1}} \neq 0$, then $m_{1, j_{1}}$ divides $m_{1, j}$ and $t_{j} / t_{j_{1}}=m_{1, j} / m_{1, j_{1}}$.

Proof. Let $j \geq j_{1}$. Since $m_{1, j_{1}} \neq 0$, we can write $m_{1, j}=q m_{1, j_{1}}+r$ where $q, r \in \mathbb{Z}$ with $0 \leq r<\left|m_{1, j_{1}}\right|$. Now

$$
w^{t_{j}-q t_{j_{1}}} \equiv u_{1}^{r} u_{2}^{m_{2, j}-q m_{2, j_{1}}} \ldots u_{k}^{m_{k, j}-q m_{k, j_{1}}} \quad\left(\bmod [U, H]^{H} \gamma_{j_{1}}(H)\right) .
$$

This contradicts the minimality of $\left(*_{j_{1}}\right)$ unless $t_{j}=q t_{j_{1}}$. Hence

$$
1 \equiv u_{1}^{r} u_{2}^{m_{2, j}-q m_{2, j_{1}}} \ldots u_{k}^{m_{k, j}-q m_{k, j_{1}}} \quad\left(\bmod [U, H]^{H} \gamma_{j_{1}}(H)\right) .
$$

If $r \neq 0$, then

$$
u_{1}^{r} \equiv u_{2}^{q m_{2, j_{1}}-m_{2, j}} \ldots u_{k}^{q m_{k, j_{1}}-m_{k, j}} \quad\left(\bmod [U, H]^{H} \gamma_{j_{1}}(H)\right) .
$$

Raising both sides of $\left(*_{j_{1}}\right)$ to the $r^{t h}$-power and substituting the above gives a $\left(*_{j_{1}}\right)$ representation of a positive power of $w$ with the exponent on $u_{1}$ being $0<\left|m_{1, j_{1}}\right|$. This contradicts the minimality of $\left(*_{j_{1}}\right)$. Thus $r=0$ and we have $m_{1, j} / m_{1, j_{1}}=$ $q=t_{j} / t_{j_{1}}$. 
The claim also implies that $m_{1, j} \neq 0$ if $j \geq j_{1}$ and $m_{1, j_{1}} \neq 0$ (since $t_{j} \in \mathbb{Z}_{+}$).

Either $m_{1, j}=0$ for all $j \in \mathbb{Z}_{+}$or there is a least $j_{1} \in \mathbb{Z}_{+}$with $m_{1, j_{1}} \neq 0$. Then for all $j \geq j_{1}$, there are $q_{j} \in \mathbb{Z} \backslash\{0\}$ such that the minimal $j^{t h}$-representation for all positive powers of $w$ is

$$
w^{t_{j_{1}} q_{j}} \equiv u_{1}^{m_{1, j_{1}} q_{j}} u_{2}^{m_{2, j}} \ldots u_{k}^{m_{k, j}} \quad\left(\bmod [U, H]^{H} \gamma_{j}(H)\right) .
$$

Let $w_{1}=w^{t_{j_{1}}} u_{1}^{-m_{1, j_{1}}}$ if such a $j_{1}$ exists; if no such $j_{1}$ exists, let $j_{1}=1$ and $w_{1}=w^{t_{1}}=w^{t_{1}} u_{1}^{-m_{1,1}}$.

Repeating the above argument with $w_{1}$ in place of $w$, we can find $j_{2} \geq j_{1}$ and $q_{j}^{\prime} \in \mathbb{Z}$ such that

$$
w_{1}^{t_{j 2}^{\prime} q_{j}^{\prime}} \equiv u_{2}^{m_{2, j_{2}}^{\prime} q_{j}^{\prime}} u_{3}^{m_{3, j}^{\prime}} \ldots u_{k}^{m_{k, j}^{\prime}} \quad\left(\bmod [U, H]^{H} \gamma_{j}(H)\right)
$$

for all $j \geq j_{2}$.

Let $w_{2}=w_{1}^{t_{j_{2}}^{\prime}} u_{2}^{-m_{2, j_{2}}^{\prime}}$.

Continuing in this way, we obtain $m_{1}, \ldots, m_{k} \in \mathbb{Z}$ and $m_{0}, q_{j} \in \mathbb{Z}_{+}$such that

$$
\left(w^{m_{0}} u_{1}^{m_{1}} \cdots u_{k}^{m_{k}}\right)^{q_{j}} \in[U, H]^{H} \gamma_{j}(H)
$$

for all sufficiently large $j \in \mathbb{Z}_{+}$. This completes the proof of the lemma.

We now prove Lemma 2.4.

Proof. Assume the hypotheses of the lemma. Let $G$ be a nilpotent class $c$ ogroup and $g_{1}, \ldots, g_{n} \in G$. Let $\varphi: F \rightarrow G_{1}=\left\langle g_{1}, \ldots, g_{n}\right\rangle$ be determined by $x_{i} \mapsto g_{i}(1 \leq i \leq n)$. Let $v=v\left(g_{1}, \ldots, g_{n}\right)=\bigvee_{j=1}^{k}\left|u_{j}\left(g_{1}, \ldots, g_{n}\right)\right|$, and $C_{v}$ be its value in $G$. Hence $u_{1}\left(g_{1}, \ldots, g_{n}\right), \ldots, u_{k}\left(g_{1}, \ldots, g_{n}\right) \in C(v)$. By Lemma 1.4. $U^{\varphi} \subseteq C(v)$, the cover of $C_{v}$ in $G$. Since $G$ is weakly Abelian, $U^{G} \subseteq C(v)$; thus $\left([U, F]^{F}\right)^{\varphi}=\left[U^{G_{1}}, G_{1}\right] \subseteq[C(v), G] \subseteq C_{v}$. So if $w\left(x_{1}, \ldots, x_{n}\right) \in \Gamma_{[U, F]}(F)$, then $w\left(x_{1}, \ldots, x_{n}\right)^{m} \in[U, F]^{F} \gamma_{c+1}(F)$ for some $m=m(c) \in \mathbb{Z}_{+}$. Thus $w\left(g_{1}, \ldots, g_{n}\right)^{m} \in$ $C_{v}$. Therefore $w\left(g_{1}, \ldots, g_{n}\right) \in C_{v}$ (convex subgroups are isolated). Consequently

$$
\left|w\left(g_{1}, \ldots, g_{n}\right)\right| \ll \bigvee_{j=1}^{k}\left|u_{j}\left(g_{1}, \ldots, g_{n}\right)\right|
$$

Since $g_{1}, \ldots, g_{n}$ were arbitrary in $G$, we get that $(* *)$ holds in $G$. Hence $(* *)$ holds in all nilpotent o-groups (it is independent of the nilpotency class of $G$ ), and so in $\hat{\mathfrak{N}}$.

We now have, for example, by Theorem D:

Corollary 2.5. If a lattice-ordered group $G \in \hat{\mathfrak{N}}$ is finitely presented as an abstract group, then $G$ is residually torsion-free-nilpotent.

Finally in this section, we can now exhibit a central order on a free group that does not belong to $\hat{\mathfrak{N}}$; cf., $[\underline{5}$.

Corollary 2.6. There is a central order on $F_{4}$, the free group on 4 generators, so that $\left(F_{4}, \leq\right) \notin \hat{\mathfrak{N}}$.

Proof. Let $K_{0}$ be the centrally ordered Kopytov group of Example 2.2. and let $a, b, c, \beta$ be the free generators of $F_{4}$. Let $\theta: F_{4} \rightarrow K_{0}$ be the homomorphism given by $a \mapsto a, b \mapsto b, c \mapsto c_{0,0}$, and $\beta \mapsto \beta$. Let $C=\operatorname{ker}(\theta)$. Then $C$ is a free group and so can be centrally ordered using the series $C \cap \gamma_{j}\left(F_{4}\right)\left(j \in \mathbb{Z}_{+}\right)$. Then $F_{4}$ is 
centrally ordered by $f>1$ iff either $\left(f \notin C\right.$ and $\left.f^{\theta} \in\left(K_{0}\right)_{+}\right)$or $f \in C_{+}$. Since $K_{0} \notin \hat{\mathfrak{N}}$, we have $\left(F_{4}, \leq\right) \notin \hat{\mathfrak{N}}$.

\section{IDENTITIES IN NILPOTENT $\ell$-GROUPS}

In Lemma 2.4, we found a set of identities that hold in $\hat{\mathfrak{N}}$. We now show that these are sufficient to define $\hat{\mathfrak{N}}$.

Let $\tilde{\mathfrak{N}}$ be the variety of $\ell$-groups defined by the identities $(* *)$ in Lemma 2.4 , That is, the defining identities for $\tilde{\mathfrak{N}}$ are:

for each $k, m, n \in \mathbb{Z}_{+}, u_{1}\left(x_{1}, \ldots, x_{n}\right), \ldots, u_{k}\left(x_{1}, \ldots, x_{n}\right) \in F=F\left(x_{1}, \ldots, x_{n}\right)$ and $w\left(x_{1}, \ldots, x_{n}\right) \in \Gamma_{[U, F]}(F)$,

$$
\left|w\left(x_{1}, \ldots, x_{n}\right)\right|^{m} \leq \bigvee_{j=1}^{k}\left|u_{j}\left(x_{1}, \ldots, x_{n}\right)\right|,
$$

where $U=\left\langle u_{1}\left(x_{1}, \ldots, x_{n}\right), \ldots, u_{k}\left(x_{1}, \ldots, x_{n}\right)\right\rangle$.

By Lemma 2.4.

$$
\hat{\mathfrak{N}} \subseteq \tilde{\mathfrak{N}} \subseteq \mathcal{W} \subseteq \mathcal{R}
$$

For, putting $k=1, n=2, u_{1}=x_{1}$, and $w=\left[x_{1}, x_{2}\right] \in\left[\left\langle x_{1}\right\rangle, F\right] \subseteq \Gamma_{\left[\left\langle x_{1}\right\rangle, F\right]}(F)$, we obtain the identities $\left|\left[x_{1}, x_{2}\right]\right|^{m} \leq\left|x_{1}\right|$ for all $m \in \mathbb{Z}_{+}$which define the weakly Abelian variety by Lemma 1.4 .

The proof of Theorem $\mathrm{D}$ from Lemmata 2.3 and 2.4 (see Section 2) applies equally to $\tilde{\mathfrak{N}}$; that is,

Lemma 3.1. Let $G_{0}$ be a lattice-ordered group and $G_{0} \in \tilde{\mathfrak{N}}$. If there is a group homomorphism $\varphi$ from a finitely presented group $G$ into $G_{0}$, then $\Gamma(G) \subseteq \operatorname{ker}(\varphi)$.

The main result of this section is

Theorem E. $q(\mathfrak{N})=\hat{\mathfrak{N}}=\tilde{\mathfrak{N}}$.

Since we have provided an explicit set of defining laws for $\tilde{\mathfrak{N}}$, Theorem $\mathrm{E}$ gives both Theorem $B$ and a set of defining identities for $\hat{\mathfrak{N}}$.

To prove the theorem, we will need one extra technical fact.

Lemma 3.2. Let $F=F\left(x_{1}, \ldots, x_{n}\right)$ be a free group of rank $n$ and $H=\left\langle h_{1}, \ldots, h_{n}\right.$ : $\left.u_{1}\left(h_{1}, \ldots, h_{n}\right)=1, \ldots, u_{p}\left(h_{1}, \ldots, h_{n}\right)=1\right\rangle$. Let $G \in \tilde{\mathfrak{N}}$ be an o-group that is a homomorphic image of $H$ (qua group), say, $G=H^{\varphi}$ with $g_{i}=h_{i}^{\varphi}(i=1, \ldots, n)$. Let $\left\{w_{1}\left(x_{1}, \ldots, x_{n}\right), \ldots, w_{k}\left(x_{1}, \ldots, x_{n}\right)\right\} \subseteq F$ be such that $\left\{w_{1}\left(g_{1}, \ldots, g_{n}\right), \ldots\right.$, $\left.w_{k}\left(g_{1}, \ldots, g_{n}\right)\right\} \subseteq G_{+}$. Then there exists $j_{0} \in \mathbb{Z}_{+}$and a total order on $H / I_{j_{0}}(H)$ such that

$$
\left\{I_{j_{0}}(H) w_{1}\left(h_{1}, \ldots, h_{n}\right), \ldots, I_{j_{0}}(H) w_{k}\left(h_{1}, \ldots, h_{n}\right)\right\} \subseteq\left(H / I_{j_{0}}(H)\right)_{+} .
$$

We now deduce Theorem E from Lemma 3.2 .

Proof. By Lemma 2.4 it suffices to show that $\tilde{\mathfrak{N}} \subseteq q(\mathfrak{N})$. Since $\tilde{\mathfrak{N}} \subseteq \mathcal{R}$, by Lemma 1.3 it is enough to prove that every finitely generated o-group $G \in \tilde{\mathfrak{N}}$ belongs to $q(\mathfrak{N})$.

Let $G$ be such an o-group generated by $g_{1}, \ldots, g_{n}$. Let $F$ be the free group on $x_{1}, \ldots, x_{n}$. Let $\left\{R_{i}\left(x_{1}, \ldots, x_{n}\right): i \in \mathbb{Z}_{+}\right\}$be the set of all words in $F$ such that $R_{i}\left(g_{1}, \ldots, g_{n}\right)=1$ in $G$. Then $G \cong F / K$, where $K=\left\langle R_{i}\left(x_{1}, \ldots, x_{n}\right): i \in \mathbb{Z}_{+}\right\rangle^{F}$. 
Let $\varphi: F \rightarrow G$ be the group homomorphism determined by $\varphi: x_{\ell} \mapsto g_{\ell}(\ell=$ $1, \ldots, n)$. Let $\left\{c_{i}\left(g_{1}, \ldots, g_{n}\right): i \in \mathbb{Z}_{+}\right\}$be an enumeration of $G_{+}$.

For each $m \in \mathbb{Z}_{+}$, let $K_{m}=\left\langle R_{i}\left(x_{1}, \ldots, x_{n}\right): 1 \leq i \leq m\right\rangle^{F}$, and $G_{m} \cong F / K_{m}$; so $G_{m}=F^{\varphi_{m}}$, say. By construction, each $G_{m}$ is a finitely presented group and the o-group $G$ is a group homomorphic image of it.

By Lemma 3.2, for each $m \in \mathbb{Z}_{+}$there is $j=j(m) \in \mathbb{Z}_{+}$and a total order on the torsion-free nilpotent group $N_{m, j(m)}:=G_{m} / I_{j(m)}\left(G_{m}\right)$ such that elements

$$
I_{j(m)}\left(G_{m}\right) c_{1}\left(x_{1}^{\varphi_{m}}, \ldots, x_{n}^{\varphi_{m}}\right), \ldots, I_{j(m)}\left(G_{m}\right) c_{m}\left(x_{1}^{\varphi_{m}}, \ldots, x_{n}^{\varphi_{m}}\right)
$$

all belong to $\left(N_{m, j(m)}\right)_{+}$.

Let $\mathcal{U}$ be an ultrafilter on $\mathbb{Z}_{+}$, and let $H=\left(\prod_{m \in \mathbb{Z}_{+}} N_{m, j(m)}\right) / \mathcal{U}$ be the resulting ultraproduct. Since each $N_{m, j(m)}$ is an o-group, so is $H$. Moreover, since each $N_{m, j(m)}$ is nilpotent, we get $H \in q(\mathfrak{N})$. Then $G$ can be mapped into $H$ by mapping $g_{\ell}$ to the $\mathcal{U}$-equivalence class of the element whose $m^{t h}$ coordinate is $I_{j(m)}\left(G_{m}\right) x_{\ell}^{\varphi_{m}}$ $\left(m \in \mathbb{Z}_{+}\right)$, where $\ell=1, \ldots, n$. Call this map $\theta$. It is well defined since for each $i \in \mathbb{Z}_{+},\left\{m \in \mathbb{Z}_{+}: i \leq m\right\} \in \mathcal{U}$ (so $\left.R_{i}\left(g_{1}^{\theta}, \ldots, g_{n}^{\theta}\right)=1\right)$. Thus $\theta$ is a group homomorphism that preserves order since $\left\{c_{i}\left(g_{1}, \ldots, g_{n}\right): i \in \mathbb{Z}_{+}\right\}$is an enumeration of $G_{+}$(again use $\left\{m \in \mathbb{Z}_{+}: i \leq m\right\} \in \mathcal{U}$ ). Hence $G$ is $\ell$-isomorphic to a (sublattice) subgroup of the o-group $H \in q(\mathfrak{N})$. Consequently, $G \in q(\mathfrak{N})$.

It only remains to prove Lemma 3.2 .

Proof. If the lemma were false, choose a counter-example $G$ with $k$ minimal. By the minimality and rechristening, we may assume that

(I) $w_{1}\left(g_{1}, \ldots, g_{n}\right)>\cdots>w_{k}\left(g_{1}, \ldots, g_{n}\right)>1$ in $G$, and

(II) there is $j_{0} \in \mathbb{Z}_{+}$and a total order on $H / I_{j_{0}}(H)$ in which

$$
I_{j_{0}}(H) w_{2}\left(h_{1}, \ldots, h_{n}\right), \ldots, I_{j_{0}}(H) w_{k}\left(h_{1}, \ldots, h_{n}\right) \in\left(H / I_{j_{0}}(H)\right)_{+} .
$$

Whenever $j \geq j_{0}$, there is a natural homomorphism from $H / I_{j}(H)$ onto $H / I_{j_{0}}(H)$ determined by $I_{j}(H) h \mapsto I_{j_{0}}(H) h$. Since $H / I_{j}(H)$ is a torsion-free nilpotent group, by Lemma 1.2 we can lift any total order from $H / I_{j_{0}}(H)$ to $H / I_{j}(H)$ (let $I_{j}(H) h \in$ $\left(H / I_{j}(H)\right)_{+}$iff $I_{j_{0}}(H) h \in\left(H / I_{j_{0}}(H)\right)_{+}$; extend this partial order to a total order on $H / I_{j}(H)$ by Lemma 1.2) .

By Lemma 1.1] if $j \geq j_{0}$, we may assume that $b_{2}, \ldots, b_{k} \in\left(H / I_{j}(H)\right)_{+}$but

$$
1 \in N_{H / I_{j}(H)}\left(b_{1}, \ldots, b_{k}\right),
$$

where $b_{i}=I_{j}(H) w_{i}\left(h_{1}, \ldots, h_{n}\right)(i=1, \ldots, k)$.

Let $d_{i}=w_{i}\left(g_{1}, \ldots, g_{n}\right) \in G_{+}(i=1, \ldots, k)$, and $C=C_{d_{1}}$ be the value of $d_{1}$ in $G$. By (I) we have $d_{1}, \ldots, d_{k} \in C\left(d_{1}\right)$ and $C\left(d_{1}\right) / C \subseteq \zeta(G / C)$.

Let $K=\left\langle\left[b_{i}, h_{\ell}\right]: i=1, \ldots, k, \ell=1, \ldots, n\right\rangle^{H}$. Let $\bar{H}=H / K$ and $\bar{G}=G / C$; write $\bar{h}$ for $K h$ and $\bar{g}$ for $C g(h \in H ; g \in G)$. Since $\bar{d}_{i} \in \zeta(\bar{G})(i=1, \ldots, k), \bar{G}$ is a homomorphic image of $\bar{H}$ under the naturally induced map, say, $\bar{G}=\bar{H}^{\bar{\varphi}}$. Note that $\bar{H}$ is a finitely presented group and $\bar{G} \in \tilde{\mathfrak{N}}$.

Now $\bar{d}_{1} \geq \cdots \geq \bar{d}_{k} \geq 1$ with $\bar{d}_{1} \in \bar{G}_{+}$. Since $\bar{b}_{i} \in \zeta(\bar{H})(i=1, \ldots, k)$ and $1 \in N_{H / I_{j}(H)}\left(b_{1}, \ldots, b_{k}\right)$ for all $j \geq j_{0} \in \mathbb{Z}_{+}$, we have (for such $j$ )

$$
\bar{b}_{1}^{m_{1, j}} \cdots \bar{b}_{k}^{m_{k, j}} \equiv 1 \quad\left(\bmod I_{j}(\bar{H})\right)
$$

with $m_{1, j} \in \mathbb{Z}_{+}$and $m_{2, j}, \ldots, m_{k, j} \in \mathbb{N}$.

For each $j \geq j_{0}$, we define $\operatorname{rank}_{j}\left(\bar{b}_{1}, \ldots, \bar{b}_{k}\right)$ as the minimum of $\mid\{i \in\{1, \ldots, k\}$ : $\left.m_{i, j} \neq 0\right\} \mid$ ranging over all equivalences that can occur in (20). 
If $j \geq j_{0}$, the natural homomorphism from $\bar{H} / I_{j}(\bar{H})$ onto $\bar{H} / I_{j_{0}}(\bar{H})$ gives that $\bar{b}_{1}^{m_{1, j}} \cdots \bar{b}_{k}^{m_{k, j}} \equiv 1\left(\bmod I_{j_{0}}(\bar{H})\right)$ whenever $\bar{b}_{1}^{m_{1, j}} \cdots \bar{b}_{k}^{m_{k, j}} \equiv 1\left(\bmod I_{j}(\bar{H})\right)$. Hence $\operatorname{rank}_{j}\left(\bar{b}_{1}, \ldots, \bar{b}_{k}\right)$ is an increasing positive-integer-valued function of $j$, bounded above by $k$. Thus there is $j_{1} \geq j_{0}$ such that $\operatorname{rank}_{j}\left(\bar{b}_{1}, \ldots, \bar{b}_{k}\right)$ is constant on all integers greater than or equal to $j_{1}$, with $m_{i, j}=0$ iff $m_{i, j_{1}}=0(i=1, \ldots, k)$. By the minimality of $k$, we have that $\operatorname{rank}_{j}\left(\bar{b}_{1}, \ldots, \bar{b}_{k}\right)=k$ for all $j \geq j_{1}$. Thus, we can assume that if any of $m_{1, j}^{\prime}, \ldots, m_{k, j}^{\prime}$ is 0 , then $\bar{b}_{1}^{m_{1, j}^{\prime}}, \ldots, \bar{b}_{k}^{m_{k, j}^{\prime}} \not \equiv 1$ in (20) $\left(j \geq j_{1}\right)$.

So $m_{1, j}, \ldots, m_{k, j} \in \mathbb{Z}_{+}$for all $j \geq j_{1}$.

By (20), $\bar{b}_{1} \in \Gamma_{\bar{B}_{1}}(\bar{H})$, where $\bar{B}_{1}=\left\langle\bar{b}_{2}, \ldots, \bar{b}_{k}\right\rangle \subseteq \bar{H}$.

By Lemma 2.3 there exist $t_{1}, t_{2}, \ldots, t_{k} \in \mathbb{Z}$ with $t_{1} \in \mathbb{Z}_{+}$such that

$$
\hat{b}_{1}:=\bar{b}_{1}^{t_{1}} \bar{b}_{2}^{t_{2}} \cdots \bar{b}_{k}^{t_{k}} \in \Gamma_{\left[\bar{B}_{1}, \bar{H}\right]}(\bar{H}) .
$$

But $\left[\bar{b}_{i}, \bar{H}\right]=1$ for $i=1, \ldots, k$ by the definition of $K$. Hence $\hat{b}_{1} \in \Gamma(\bar{H})$. Therefore, for all $j \geq j_{1}$,

$$
\bar{b}_{1}^{t_{1}} \bar{b}_{2}^{t_{2}} \cdots \bar{b}_{k}^{t_{k}} \equiv 1 \quad\left(\bmod I_{j}(\bar{H})\right) .
$$

We now use that $\operatorname{rank}_{j}\left(\bar{b}_{1}, \ldots, \bar{b}_{k}\right)=k$ for all $j \geq j_{1}$ to get a contradiction if $t_{i}<0$ for some $i=2, \ldots, k$.

For reductio ad absurdum, assume that $t_{i}<0$ for some $i=2, \ldots, k$. Choose $r \leq k$ maximal so that there are $t_{i_{2}}, \ldots, t_{i_{r}}<0$, where $i_{2}, \ldots, i_{r}$ are distinct members of $\{2, \ldots, k\}$. Fix $j \geq j_{1}$. By rechristening, we may assume that $-t_{i_{2}} / m_{i_{2}, j}=$ $\max \left\{-t_{i_{2}} / m_{i_{2}, j}, \ldots,-t_{i_{r}} / m_{i_{r}, j}\right\}$. By (20) and (21), we get

$$
\bar{b}_{1}^{-t_{i_{2}} m_{1, j}+t_{1} m_{i_{2}, j}} \bar{b}_{i_{2}}^{-t_{i_{2}} m_{i_{2}, j}+t_{i_{2}} m_{i_{2}, j}} \ldots \bar{b}_{k}^{-t_{i_{2}} m_{k, j}+t_{k} m_{i_{2}, j}} \equiv 1 \quad\left(\bmod I_{j}(\bar{H})\right) .
$$

In this expression, all exponents are non-negative by the maximality of the ratio, and $-t_{i_{2}} m_{i_{2}, j}+t_{i_{2}} m_{i_{2}, j}=0<-t_{i_{2}} m_{1, j}+t_{1} m_{i_{2}, j}$. This contradicts that $\operatorname{rank}_{j}\left(\bar{b}_{1}, \ldots, \bar{b}_{k}\right)=k$. Thus $t_{1}, \ldots, t_{k} \in \mathbb{Z}_{+}$.

But $\bar{G}=\bar{H}^{\bar{\varphi}}$ and $\bar{G} \in \tilde{\mathfrak{N}}$, so $\hat{b}_{1} \in \operatorname{ker}(\bar{\varphi})$ by Lemma 3.1. Consequently,

$$
1=\hat{b}_{1}^{\bar{\varphi}}=\left(\bar{b}_{1}^{t_{1}} \bar{b}_{2}^{t_{2}} \cdots \bar{b}_{k}^{t_{k}}\right)^{\bar{\varphi}}=\bar{d}_{1}^{t_{1}} \cdots \bar{d}_{k}^{t_{k}}>\bar{d}_{1} .
$$

This is impossible as $\bar{d}_{1} \in \bar{G}_{+}$. The proof of the lemma (and hence Theorem $\mathbb{B}$ ) is now complete.

\section{The proof of Theorem $\mathrm{C}$}

We begin with a lemma.

Lemma 4.1. Let $G$ be a centrally ordered group. Suppose that $G / C$ is residually torsion-free-nilpotent for each value $C$ of $G$. Then $G \in q(\mathfrak{N})$.

Proof. Suppose that $G \notin q(\mathfrak{N})$. Then $G \notin \tilde{\mathfrak{N}}$ by Theorem $\mathrm{E}$, and so one of the identities $(* *)$ fails in $G$. Thus there are $k, m, n \in \mathbb{Z}_{+}, g_{1}, \ldots, g_{n} \in G$, $u_{1}\left(x_{1}, \ldots, x_{n}\right), \ldots, u_{k}\left(x_{1}, \ldots, x_{n}\right) \in F=F\left(x_{1}, \ldots, x_{n}\right)$, and $w\left(x_{1}, \ldots, x_{n}\right) \in$ $\Gamma_{[U, F]}(F)$ such that

$$
\left|w\left(g_{1}, \ldots, g_{n}\right)\right|^{m}>\bigvee_{j=1}^{k}\left|u_{j}\left(g_{1}, \ldots, g_{n}\right)\right|,
$$

where $U:=\left\langle u_{1}\left(x_{1}, \ldots, x_{n}\right), \ldots, u_{k}\left(x_{1}, \ldots, x_{n}\right)\right\rangle$. 
Let $C=C_{w}$ be the value of $w=w\left(g_{1}, \ldots, g_{n}\right)$ in $G$, and $H=G / C$. Let $\bar{g}:=C g(g \in G), \bar{v}=C v\left(g_{1}, \ldots, g_{n}\right)(v \in F)$, and $\bar{U}=\left\langle\bar{u}_{1}, \ldots, \bar{u}_{k}\right\rangle \subseteq H$. Now $C(w) / C \subseteq \zeta(H)$, so $\bar{w}, \bar{u}_{j} \in \zeta(H)(j=1, \ldots, k)$. It follows that $[\bar{U}, H]=1$. Since $w\left(x_{1}, \ldots, x_{n}\right) \in \Gamma_{[U, F]}(F)$, we get $\bar{w} \in \Gamma_{[\bar{U}, H]}(H)=\Gamma(H)$. This contradicts that $H$ is residually torsion-free-nilpotent.

This is enough to prove Theorem $\mathrm{C}$.

Proof. Since weakly Abelian $\ell$-groups are residually ordered, it is enough to prove the theorem for finitely generated centrally ordered Abelian-by-nilpotent groups by Lemma 1.3. The result follows from Lemmata 1.5 and 4.1

Corollary 4.2. (i) There is a weakly Abelian lattice-ordered group with the maximal condition on normal subgroups that does not belong to $\hat{\mathfrak{N}}$.

(ii) $\hat{\mathfrak{N}}$ is not closed under central extensions.

Proof. Since Example 2.2 is cyclic-by-(finitely generated metabelian), it satisfies the maximal condition on normal subgroups. (ii) follows from Example 2.2 and Theorem $\mathrm{C}$.

\section{Concluding Remarks}

The results we have obtained beg several questions. The easiest to state are:

1. Does $\hat{\mathfrak{N}}$ have a finite set of defining identities? We suspect not.

2. What varieties of lattice-ordered groups can occur between $\hat{\mathfrak{N}}$ and $\mathcal{W}$ ? Is it possible that the latter covers the former (in which case the answer to Kopytov's question is only just "no")?

Added in proof. The results in Section 3 can be used to obtain the converse of Theorem D. Consequently, $q(\mathfrak{N})$ can be defined by group implications and contains the same groups as the quasi-variety generated by all torsion-free nilpotent groups.

\section{ACKNOWLEDGEMENTS}

The first author would like to thank Queens' College, Cambridge and the Russian Foundation for Basic Research (grant no. 03-01-00320) for funding his visit to Cambridge (9/25/03-10/10/03). He is most grateful to the College and DPMMS for their help and hospitality while he was visiting. The second author wishes to thank John S. Wilson for several discussions that resulted in the simplification of the proof of (i) (in Section 2) presented here.

\section{REFERENCES}

[1] V. V. Bludov, On locally nilpotent groups, Trudy Inst. Mat. Sobolev 30 (1996), 26-47 (in Russian): English translation, Siberian Advances in Math. 8 (1998) 49-79. MR.1651902 (99m:20074)

[2] V. V. Bludov, A. M. W. Glass and A. H. Rhemtulla, Ordered groups in which all convex jumps are central, J. Korean Math. Soc. 40 (2003), 225-239. MR1958028 (2003j:06020)

[3] V. V. Bludov, A. M. W. Glass and A. H. Rhemtulla, On centrally ordered groups, J. Algebra 291 (2005), 129-143. MR.2158514 (2006h:20053)

[4] A. M. W. Glass, Partially Ordered Groups, World Scientific Pub. Co., Singapore, 1999. MR1791008 (2001g:06002)

[5] A. M. W. Glass, Weakly Abelian lattice-ordered groups, Proc. American Math. Soc. 129 (2001), 677-684; corrigendum: ibid 130 (2001), 925-926. MR.1801994(2002a:06022) 
[6] S. A. Gurchenkov, On varieties of weakly Abelian $\ell$-groups, Mat. Slovaca 42 (1992), 437-441. MR.1195037 (94a:20067)

[7] P. Hall, Nilpotent Groups, Lectures given at the Canadian Mathematical Congress, University of Alberta, 1957.

[8] V. M. Kopytov, Lattice-ordered locally nilpotent groups, Algebra i Logika 14 (1975), 407413 (in Russian); English translation Algebra and Logic 14 (1975), 249-251. MR0401583 (53:5410)

[9] V. M. Kopytov and N. Ya. Medvedev, The Theory of Lattice-ordered Groups, Kluwer Acad. Pub., Dordrecht, 1994. MR 1369091 (97k:06028)

[10] Kourovka Notebook, Unsolved Problems in Group Theory, eds. V. D. Mazurov and E. I. Khukhro, 13th edition, Novosibirsk, 1995. MR1392713 (97d:20001)

[11] J. Martinez, Varieties of lattice-ordered groups, Math. Z. 137 (1974), 265-284. MR0354483 $(50: 6961)$

[12] R. B. Mura and A. H. Rhemtulla, Orderable Groups, Lecture Notes in Pure and Applied Maths. 27, M. Dekker, New York, 1977. MR0491396 (58:10652)

[13] N. R. Reilly, Nilpotent, weakly Abelian and Hamiltonian lattice-ordered groups, Czech. Math. J. 33 (1983), 348-353. MR0718919 (85m:06035)

[14] D. J. S. Robinson, A Course in the Theory of Groups (second edition), Graduate Texts in Math. 80, Springer-Verlag, Heidelberg, 1996. MR.1357169 (96f:20001)

[15] R. Warfield, Nilpotent Groups, Lecture Notes in Math. 513, Springer-Verlag, Berlin, 1976. MR0409661 (53:13413)

[16] The Black Swamp Problem Book is edited by W. Charles Holland (Bowling Green State University, Ohio 43403, U.S.A.) and is kept there by him (in the formerly Black Swamp region of Ohio).

Institute of Mathematics and Economics, Irkutsk State University, Irkutsk, 664003 RUSSIA

E-mail address: bludov@math.isu.ru

Department of Pure Mathematics and Mathematical Statistics, Centre for Mathematical Sciences, Wilberforce Rd., Cambridge CB3 0WB, England

E-mail address: amwg@dpmms.cam.ac.uk 